\title{
SERIES DE TIEMPO DE CLOROFILAS $a, b y c$, $Y$ SUS FEOFITINAS EN LAS BOCAS DE DOS LAGUNAS COSTERAS
}

por:

ROBERTO MILIAN NUNEEZ

$\mathrm{y}$

SAUL ALVAREZ BORREGO

Centro de Investigación Científica y de Educación Superior de Ensenada, B. C. Espinoza 843, Ensenada, B.C. México

RESUMEN.

Del 1 al 7 de febrero y del 20 de junio al 7 de julio de 1977 se realizaron muestreos cuasicontinuos en las bocas del Estero de Punta Banda y Bahía San Quintín, Baja California, respectivamente. Se generaron series de tiempo de clorofilas $a, b$ y $c$ y sus feofitinas, con intervalo de muestreo de una hora. En el Estero Ios rangos de las clorofilas $a, b$ y c fueron: 0.3 a $1.5 \mathrm{mg} \mathrm{m}^{-3}$; y 0.1 a $0.6 \mathrm{mg} \mathrm{m}^{-3} ; \mathrm{y} 0.1$ a $2.6 \mathrm{mg} \mathrm{m} \mathrm{m}^{-3}$, respectivamente. El rango de feofitina a fue 0.0 a $0.9 \mathrm{mg} \mathrm{m}^{-3}$. Las feofitinas $b$ y $c$ no se detectaron en concentraciones significativamente diferentes de cero. En San Quintín los rangos de las clorofilas a, b y c y sus feofitinas fueron: 0.8 a $15.7 \mathrm{mg} \mathrm{m} \mathrm{m}^{-3} ; 0.1$ a $2.9 \mathrm{mg} \mathrm{m}^{-3} ; 0.2$ a $10.5 \mathrm{mg} \mathrm{m} \mathrm{m}^{-3} ; 0.1$ a $11.0 \mathrm{mg} \mathrm{m}^{-3}$; 0.0 a $2.4 \mathrm{mg} \mathrm{m}^{-3} ; y^{\prime} 0.0$ a $15.0 \mathrm{mg} \mathrm{m}^{-3}$. Los valores de los diferentes pigmentos muestran en general una distribución irregular en forma de "manchas". En el Estero la clorofila a presentó una cierta correlación con las mareas, con valores altos 
correspondiendo en general con mareas altas $y$ viceversa. En San Quintin, la variacion de este pigmento mostró el efecto de la alternancia de eventos de surgencia, con valores menores durante surgencia intensa y valores mayores en períodos de relajamiento. En base a los resultados se sugiere tomar con cautela las estimaciones de productividad orgánica primaria que requieren de incubación, ya que en sólo una hora la concentración de clorofila a puede cambiar hasta por un factor de cinco hacia valores mayores o menores.

ABSTRACT.

During February 1 to 7 and June 20 to July 7 , 1977, cuasicontinuous sampling was done at the mouths of two coastal lagoons of Baja California, Estero de Punta Banda and San Quintin bay, respectively. Time series of chlorophylls $a, b$ and $c$ and their phaeopigments were generated with a sampling interval of one hour. At the mouth of the Estero, the chlorophylls $\mathrm{a}, \mathrm{b}$ and $\mathrm{c}$ ranges were: 0.3 to $1.5 \mathrm{mg} \mathrm{m} \mathrm{m}^{-3} ; 0.1$ to $0.6 \mathrm{mg} \mathrm{m}^{-3}$; and 0.1 to $2.6 \mathrm{mg} \mathrm{m}^{-3}$, respectively. The phaeophytin a range was 0.0 to $0.9 \mathrm{mg} \mathrm{m} \mathrm{m}^{-3}$. The phaeophytins $\mathrm{b}$ and $\mathrm{c}$ were not detected in concentrations significantly different from zero. At San Quintin, the ranges of chlorophylls $a, b$ and $c$ and their

phaeophytins were: 0.8 to $15.7 \mathrm{mg} \mathrm{m}^{-3} ; 0.1$ to 2.9 $\mathrm{mg} \mathrm{m} \mathrm{m}^{-3} ; 0.2$ to $10.5 \mathrm{mg} \mathrm{m}^{-3} ; 0.1$ to $11.0 \mathrm{mg} \mathrm{m}^{-3}$; 0.0 to $2.4 \mathrm{mg} \mathrm{m}^{-3}$; and 0.0 to $15.0 \mathrm{mg} \mathrm{m}^{-3}$. The variations in pigment concentrations indicates a very irregular "patchy" distribution. At the Estero, chlorophyll a showed a correlation with tides, with higher concentrations corresponding with high tides, and viceversa. At San Quintin, this pigment showed the effect of upwelling events, with lower concentrations during strong upwelling and higher concentrations during the relaxation periods. Based on the results presented here, we suggest that estimations of organic primary productivity made with incubation methods should be taken very cautiously, because chlorophyll a concentration may change by a factor of five, or so, in only one hour. 
INTRODUCCION.

En lagunas costeras existe una gran variabilidad de condiciones ambientales en función del tiempo. Alvarez Borrego, Lara Lara y Acosta Ruíz (1977) hicieron estudios de variación diurna de clorofila a, fosfatos y nitratos, en las bocas del Estero de Punta Banda y Bahía San Quintín, en primavera y verano de 1975. Pero con series de tiempo cortas, de sólo 26 horas, no es posible observar con claridad las tendencias de cambio y la posible correlación con otras variables. Por ello, decidimos hacer un muestreo relativamente largo en cada una de estas lagunas, para obtener un conjunto de datos que nos permitiera hacer un análisis estadístico más adecuado. En particular en Bahía San Quintín, si se quiere analizar el efecto de los eventos de surgencia, es necesario contar con series de tiempo del orden de tres semanas. Aquí presentaremos los resultados de las series de tiempo de clorofilas a, b y c, y sus feofitinas. Los resultados de mareas, temperatura, salinidad, oxígeno disuelto y fosfato inorgánico fueron presentados con anterioridad por Zertuche González y Alvarez Borrego (1978).

Acosta Ruíz y Alvarez Borrego (1974) y Chávez de Nishikawa y Alvarez Borrego (1974) han presentado con anterioridad descripciones de estos dos cuerpos de agua, por lo cual no es necesario repetirlo aquí. Brevemente, el Estero de Punta Banda es una laguna costera localizada a lo largo de la orilla sureste de la Bahía de Todos Santos (Fig. 1). Su boca se encuentra aproximadamente a 8 millas náuticas del mar abierto. Fuera de la Bahía de Todos Santos, al suroeste de Punta Banda se encuentra una zona de surgencias. La boca del Estero no es mayor de $150 \mathrm{~m}$ en mareas bajas. Bahía San Quintín se localiza a $200 \mathrm{~km}$ al sur de Ensenada, Baja California Norte. En el mar abierto inmediatamente al sur de la entrada de la Bahía ocurren surgencias intensas en verano (Dawson, 1951). La boca tiene un poco más de un kilómetro de ancho (Fig. 1). La profundidad media en la boca de San Quintín, en el canal principal donde se efectuó el muestreo, es $9 \mathrm{~m}$; $\mathrm{y}$ en el Estero de Punta Banda $7 \mathrm{~m}$.

MATERIALES Y METODOS.

Del 1 al 7 de febrero y del 20 de junio al 7 de julio de 1977, se realizaron muestreos cuasicontinuos en las bocas del Estero de Punta Banda y Bahía San Quintín, respectivamente (Fig. 1). Cada hora, día y noche, se tomaron muestras de agua superficial para determinación de salinidad, oxígeno disuelto, fosfato inorgánico, pigmentos fotosintéticos y biomasa de fitoplancton. Además, se determinó la temperatura del 


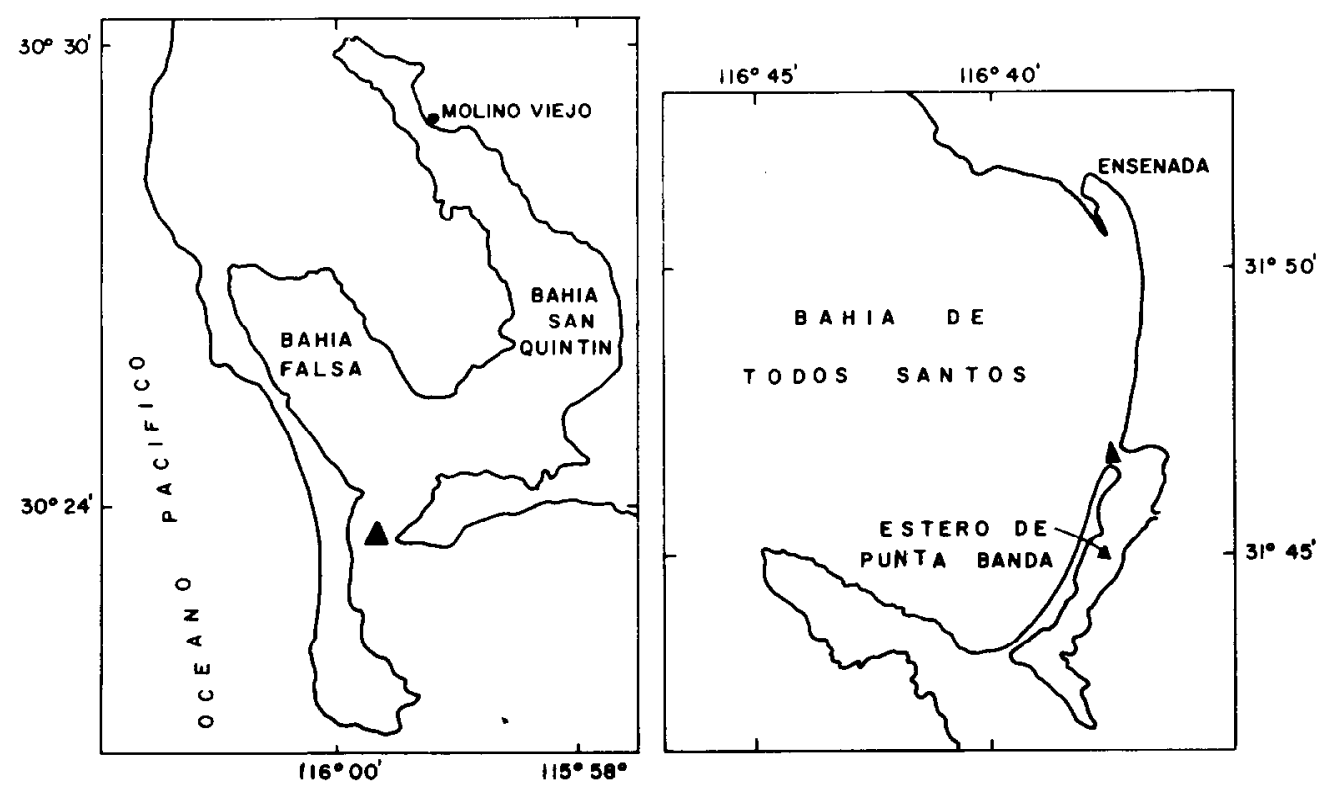

Fig. 1. Localización de los puntos de muestreo.

agua, marea y variables meteorológicas tales como temperatura del aire, irradiación solar (intensidad de luz), velocidad del viento, nubocidad y porciento de humedad. En este trabajo sólo se reportan resultados de las clorofilas a, b y c y sus feofitinas, y se discute su correlación con algunas de las otras variables.

En cada muestreo se anclaba la embarcación "SIRIUS I", de 35 pies de eslora, a manera de plataforma fija. Para el mues treo de pigmentos fotosintéticos se utilizó una botella van Dorn. Se tomaba un litro de agua de mar y se le agregaba un $\mathrm{ml}$ de una solución saturada de $\mathrm{MgCO}_{3}$. Se procedía a filtrar el agua, utilizando una bomba manual de vacío, un equipo de filtración Millipore y filtros de membrana de nitrocelulosa de $0.45 \mu$ de porosidad y $47 \mathrm{~mm}$ de diámetro. Los filtros se colocaban en discos de petri, y se congelaban hasta su análisis en el laboratorio. La extracción de los pigmentos se realizó en tubos de centrífuga de $15 \mathrm{ml}$, utilizando acetona al $90 \% \mathrm{y}$ dimetil sulfóxido en una relación de uno a uno en volumen, para mejorar la extracción (Shoaf y Lium, 1976). Se colocaban los filtros en los tubos de centrífuga, se les agregaban $5 \mathrm{ml}$ de la solución y se desintegraban con un moledor de vidrio a 1500-2000 rpm. Se lavaba el moledor con la solución y se llenaban los tubos aproximadamente a $8 \mathrm{ml}$. Se dejaban por 12 horas en extracción en un lugar fresco $\mathrm{y}$ oscuro, agitando después de las primeras 8 horas para asegurar una mejor extracción. Se aforaban a $10 \mathrm{ml}$. Se centrifugaban a 35004000 rpm durante 10 minutos. Se determinaban las absorvancias 
contra acetona al 90\% (el dimetil sulfóxido tiene las mismas propiedades espectrofotométricas que la acetona), con un espectrofotómetro marca spectronic UV 210 digital de la Shimadzu y Bausch \& Lomb. Las lecturas de absorvancia se hicieron con celdas de un centímetro, a las siguientes longitudes de onda: $750,665,645$ y 630 nanómetros, después se acidificaba la muestra con dos gotas de HCI al $10 \%$ y se leían de nuevo las absorvancias a las mismas longitudes de onda. Se hicieron correcciones por turbidez y diferencias de celda a celda, utilizando la lectura a $750 \mathrm{~nm}$. Para el cálculo de las concentraciones de los pigmentos se utilizaron las ecuaciones de Millán Núñez y Alvarez Borrego (1978).

\section{RESULTADOS.}

En el Estero de Punta Banda los rangos de las clorofilas a, b y c fueron:0.3 a $1.5 \mathrm{mg} \mathrm{m}^{-3} ; 0.1 \mathrm{a} 0.6 \mathrm{mg} \mathrm{m} \mathrm{m}^{-3} ; \mathrm{y} 0.1 \mathrm{a}$ $2.6 \mathrm{mg} \mathrm{m} \mathrm{m}^{-3}$, respectivamente. EI rango de feofitina a fue 0.0 a $0.9 \mathrm{mg} \mathrm{m}^{-3}$ (Fig. 2). No se estimaron concentraciones de feofitinas b y c significativamente diferentes de cero en el Estero. La clorofila a presentó una cierta periodicidad en el Estero, con valores relativamente altos correspondiendo en general con mareas altas, y viceversa. Las clorofilas b $\mathrm{j}$ c tuvieron una variación muy irregular, aunque muestran una cierta tendencia diurna.

En Bahía San Quintín, la clorofila a muestra cierta tendencia de variación diurna y semidiurna, quizá debido principalmente al efecto de marea, ya que los picos máximos de clorofila tienden a seguir los máximos de marea, sin tender a manifestarse en una hora particular del día lo cual implicaría una influencia más fuerte del ciclo de irradiación solar. Además, la serie de tiempo de clorofila a muestra en San Quintín una onda larga, de período aproximadamente de dos semanas, con mínimos al comienzo y al final del muestreo, $y$ valores mayores en la mitad (Fig. 3). El rango de clorofila a fue $0.8 \mathrm{a} 15.7 \mathrm{mg} \mathrm{m}$. La clorofila b no muestra tendencias claras de variación en San Quintín. Su rango fue 0.1 a 2.9 $\mathrm{mg} \mathrm{m}^{-3}$. Ia clorofila $c$ en San Quintín tuvo concentraciones similares a las de la clorofila a. Su rango fue 0.2 a 10.5 $\mathrm{mg} \mathrm{m}^{-3}$. Presentó cierta tendencia de variación diurna $\mathrm{y}$ semidiurna debida a las mareas. En general su variación fue similar a la de clorofila a, pero no se muestra con claridad la onda larga mostrada por la clorofila a. La feofitina a tuvo concentraciones relativamente altas en San Quintín (Fig. 3). Presentó cierta tendencia de variación diurna y semidiurna, correlacionada con la clorofila a en general, presentando también la onda larga con valores mayores a la mitad del período de muestreo, y menores hacia el comienzo y. 
hacia el final. Su rango fue 0.1 a $11.0 \mathrm{mg} \mathrm{m}^{-3}$. La feofitina $b$ se presentó en algunos casos con valores significativamente diferentes de cero en San Quintín, a manera de "manchas" con
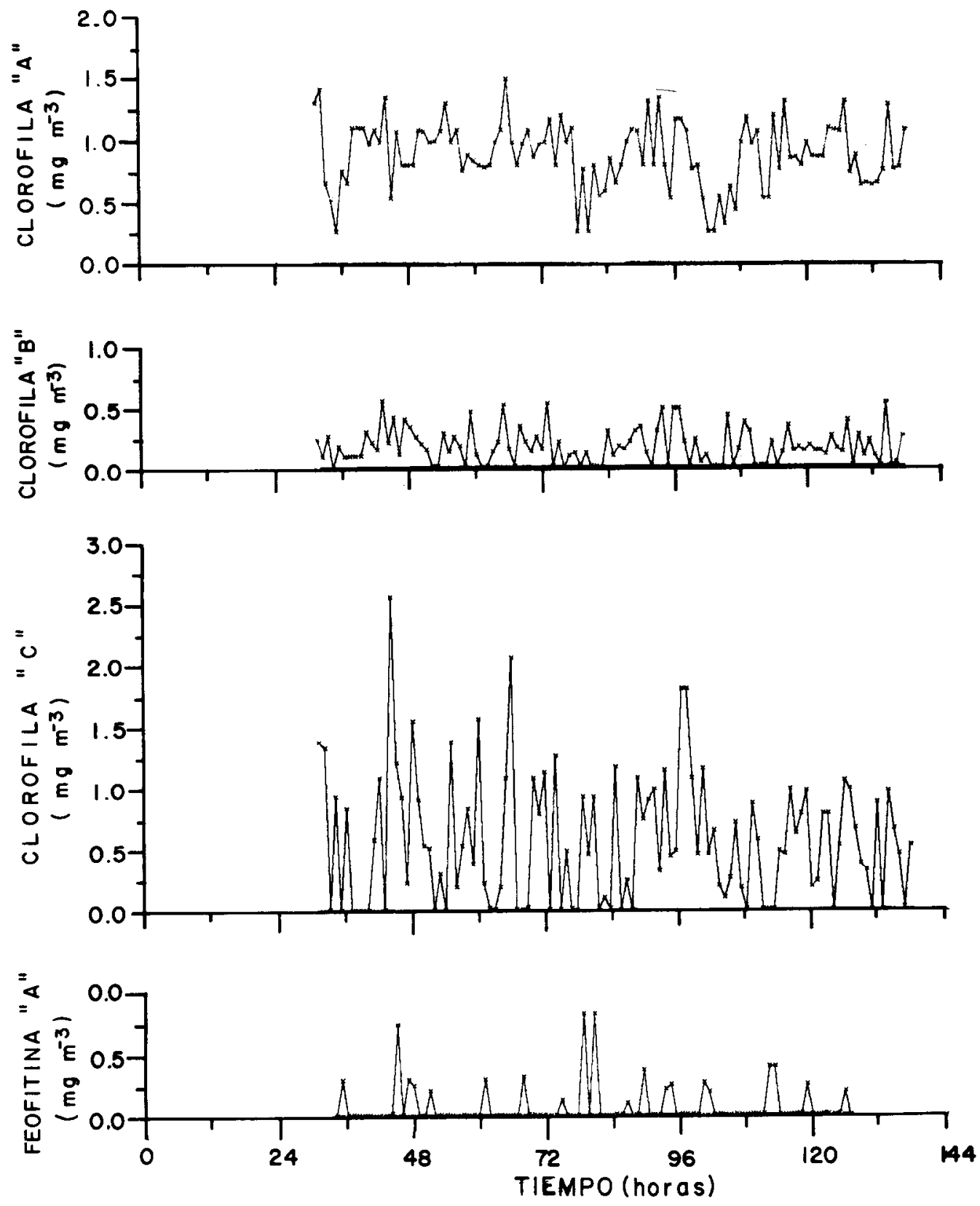

Fig. 2. Series de tiempo del Estero de Punta Banda. En la abscisa los números marcan las medias noches. 

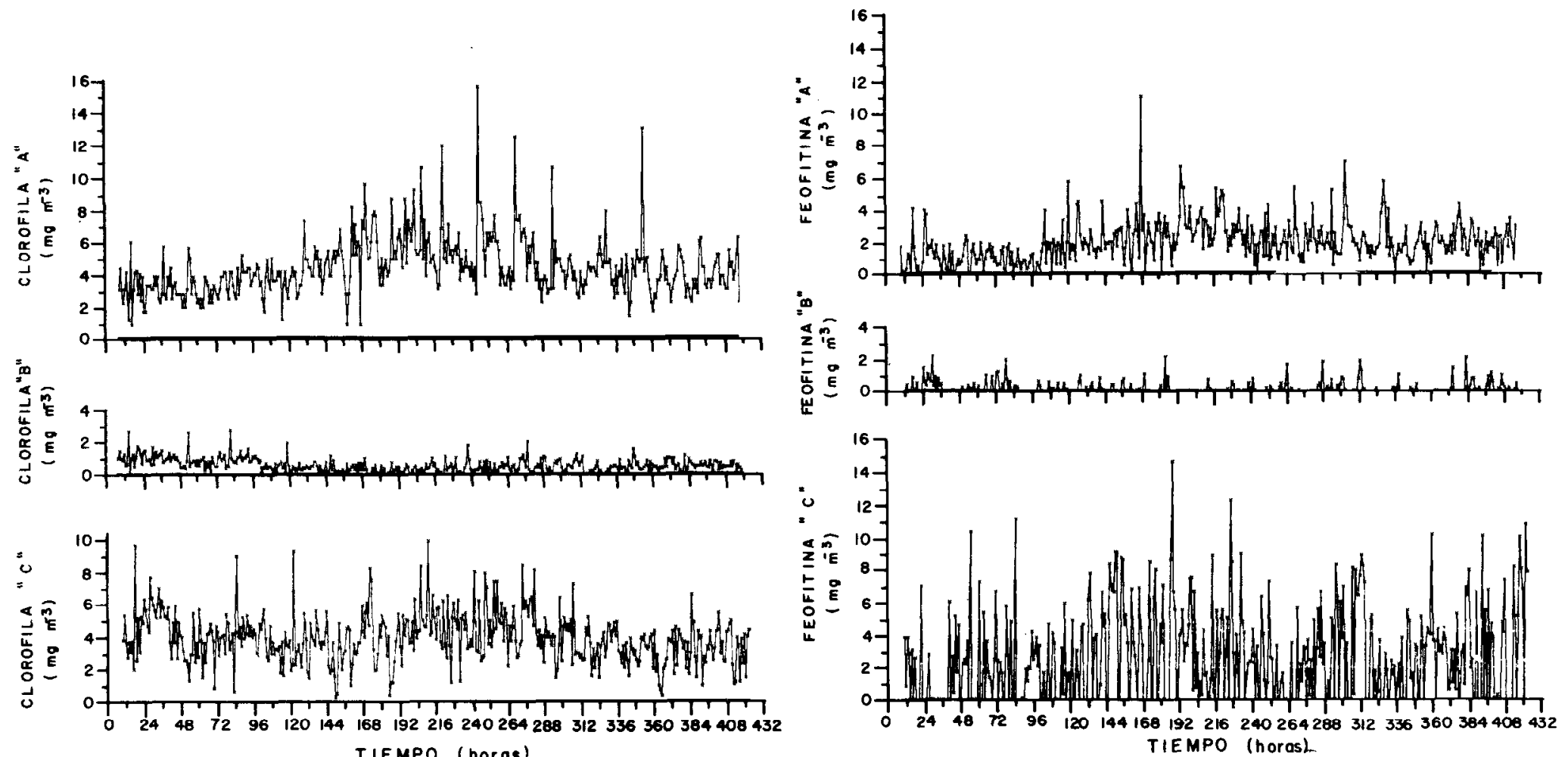
TIEMPO (horas)

Fig. 3. Series de tiempo de Bahía San Quintín. En la abscisa los numeros marcan las medias noches. 
concentraciones de hasta $2.4 \mathrm{mg} \mathrm{m}^{-3}$, sin mostrar ninguna tendencia particular en su variabilidad. La feofitina $c$ mostró una gran variabilidad, con valores desde 0.0 hasta $15.0 \mathrm{mg} \mathrm{m} \mathrm{m}^{-3}$, mostrando la distribución más marcada en forma de "manchas".

\section{DISCUSIONES.}

La concentración de pigmentos fotosintéticos, sobre todo la de clorifila a, ha sido tomada muy a menudo como un indicador de la abundancia de fitoplancton. Comparando las series de tiempo de clorofila a del Estero de Punta Banda y Bahía San quintín podemos inferir que la abundancia de fitoplancton en Bahía San Quintín puede ser hasta un orden de magnitud mayor que en el Estero. Aunque en sentido estricto las dos series no son directamente comparables ya que la del Estero es de febrero y la de San Quintín es de junio-julio. Sin embargo, Alvarez Borrego, Lara Lara y Acosta Ruíz (1977) reportaron valores máximos de sblo $3 \mathrm{mg} \mathrm{m}^{-3}$ para primavera $\mathrm{y}^{-3}$ verano en el Estero, comparados con valores reportados por ellos de hasta $13 \mathrm{mg} \mathrm{\textrm {m } ^ { - 3 }}$ para san Quintín en verano, y hasta más de $15 \mathrm{mg} \mathrm{m}^{-3}$ reportados en este trabajo. Las concentraciones de fosfato son también mayores en San Quintín que en el Estero (Alvarez Borrego, Lara Lara y Acosta Rú́z, 1977; Zertuche Conzález y Alvarez Borrego, 1978). Esto puede ser indicativo de que todos los nutrientes en general tienen mayor concentración en San Quintín. Los eventos de surgencia deben ser responsables de la mayor fertilidad en San Quintín. La boca del Estero queda por 10 menos 10 millas náuticas alejada de la surgencia de Punta Banda. El efecto de esta surgencia debe ser muy atenuado por la Bahía de Todos Santos. Las altas concentraciones de pigmentos fotosintéticos y sus productos de degradación, así como las de fosfato, en San Quintín, no pueden atribuirse a la presencia de los pastos marinos, ya que el Estero también cuenta con una densa población de pastos.

La onda larga mostrada sobre todo por la serie de tiempo de clorofila a en Bahía San Quintín, con un período del orden de dos semanas, muestra el efecto de la alternancia de eventos de surgencia. Las relativamente bajas concentraciones de clorofila a en los primeros y últimos días del muestreo, indican que el agua había aflorado recientemente y no había habido oportunidad para la proliferación del fitoplancton. Mientras que en los días intermedios, con una surgencia más débil, o período de relajamiento, el tiempo de residencia era mayor y la biomasa de fitoplancton aumentó mediante un uso más efectivo de los nutrientes. En general, con relación a esta onda larga, bajas 
concentraciones de clorofila a correspondieron a bajas temperaturas, concentraciones de oxígeno por debajo del nivel de saturación (hasta 60\%) y altas concentraciones de fosfato (Zertuche González y Alvarez Borrego, 1978), y viceversa.

Los datos indican que en general las concentraciones de pigmentos fotosintéticos en el área de las bocas de estas lagunas costeras dependen en gran medida de las condiciones imperantes en la zona océanica adyacente.

La gran variabilidad de la concentración de los pigmentos, mostrada en las series de tiempo, indica una distribución espacial del fitoplancton muy compleja, en forma de "manchas" de diversos tamaños. Es claro que en aguas como las de estas lagunas costeras, donde las corrientes de marea imponen condiciones muy dinámicas, cualquier método para estimar la productividad orgánica primaria que requiera de incubación en botellas, proporciona resultados que deben tomarse como una primera aproximación a la realidad, con muchas reservas. Ia concentración de clorofila a, y por lo tanto la de fitoplancton, cambia a veces hasta por un factor de cinco en sblo una hora. El no contar todavía con un método satisfactorio que no requiera de incubación para estimar la fotosíntesis nos obliga a utilizar métodos como el de carbono catorce. En estos casos una manera más satisfactoria sería el realizar incubaciones

seriadas, por ejemplo: tomar una muestra a las 9 de la mañana e incubarla hasta las 3 de la tarde, luego otra a las 10 de la mañana incubándola hasta las 2 de la tarde, y otra a las 11 para incubarla hasta la una, tomando finalmente el promedio. El promedio será más representativo que una muestra en particular. Otra manera sería el asumir que la fotosíntesis por unidad de clorofila es constante para el día de muestreo. Con una sola incubación y determinando la concentración de clorofila a en por lo menos cinco a diez muestras tomadas a intervalos de dos ó una hora, se tendría una estimación de la fotosíntesis más satisfactoria que la obtenida con sólo la incubación. Platt (1975) utilizó algo similar a este último método, pero para estimar la fotosíntesis en varias localidades de la cuenca Bedford, Nueva Escocia, en base a la incubación realizada en una localidad, y las determinaciones de clorifila a en todas las localidades.

La gran variabilidad de la concentración de estos pigmentos en el sentido horizontal, mostrada en nuestras series de tiempo, también ha sido observada en el sentido vertical por lo menos en la zona costera. Strickland (1968), utilizando un sistema de bombeo continuo y un fluorómetro, encontró que la clorofila a puede variar hasta por un factor de dos o más en menos de cinco metros, hacia mayores o menores valores, en 
la zona frente a La Jolla, California. Comparando sus resultados por bombeo con los obtenidos por muestreo discreto encontró que el error en este último puede ser hasta de $25 \%$ cuando se calcula el valor integrado en por unidad de área. En la boca de Bahía San Quintín, Alvarez Borrego, Lara Lara y Acosta Rú́z (1977) encontraron que, debido a la turbulencia por las corrientes de marea, la columina de agua es bastante homogénea con relación a la concentración de clorofila a.

Glooschenko, Curl y Small (1972)reportaron que las concentraciones de clorofila a en fitoplancton tienen una periodicidad diurna en aguas costeras de Oregon. Las máximas concentraciones en superficie ocurrieron a menudo alrededor de media noche. Estos mismos autores hicieron experimentos de laboratorio con Skelotonema costatum y demostraron que el ciclo diurno de clorofila a, expresado en por célula, estaba relacionado con la intensidad de la luz y la duración de exposición. El efecto del ciclo de irradiación solar no es claro en nuestras series de tiempo debido al efecto de las mareas. En el Estero de Punta Banda la serie de clorofila a mostró mínimos alrededor del mediodía y máximos alrededor del comienzo de las noches. Sin embargo, es aparente cierta correlación con las mareas, con los valores mínimos de clorofila asociados con las marea bajando. En San Quintín se presentaron máximos de clorofila a en diversas horas del día y la noche. La posible relación con el ciclo diurno de irradiación solar se presentó muy obscurecida por períodos de variación más cortos. Las otras clorofilas y sus productos de degradación tuvieron variaciones más irregulares que las de clorofila. Es muy difícil concluir sobre la relación de su variación con la de otras variables ambientales.

Gran parte de la variación en períodos cortos, de una a cinco horas, puede deberse al efecto combinado de la distribución espacial en forma de manchas y la turbulencia por las corrientes de marea. Platt (1972) y Denman y Platt (1975), al analizar algunas series de tiempo de temperatura y clorofila a, generadas en el golfo de San Lorenzo, sugirieron que en escalas de $10 \mathrm{~m} \mathrm{a} 1 \mathrm{~km}$ la abundancia local de fitoplancton puede ser controlada por los procesos físicos de transporte del agua y no por algún comportamiento dinámico de los organismos, tales como los que generarían gradientes de reproducción y fotosíntesis.

La clorofila b y su feofitina fueron las menos abundantes debido a que pocas especies contienen esta clorofila (Riley y Wilson, 1967). Las clorofilas a y c tuvieron concentraciones similares. La presencia de las feofitinas es a veces explicada en términos de decaimiento $\mathrm{y}$ degradación de las poblaciones fitoplanctónicas por encontrarse en una etapa avanzada de 
la sucesión, o bién en términos del pastoreo por zooplancton. Pero en el Estero de Punta Banda, en invierno y con menores concentraciones de nutrientes, la feofitina a se presentó pocas veces y las b y c no se presentaron en concentraciones detectables; mientras que en San Quintín, en verano y con el efecto de surgencia presente, las tres feofitinas se presentaron en concentraciones relativamente altas. Por ésto, $y$ debido a que no contamos con datos sobre abundancia y tipo de zooplancton, no podemos obtener conclusiones sobre las causas de la presencia y variabilidad de las feofitinas.

RECONOCTMIENTOS.

Agradecemos a los companteros oceanólogos Manuel de Jesús Acosta Ruíz, Gilberto Gaxiola Castro, José Rubén Lara Lara, Vidal Huitrón Barajas y Juan Manuel Rodríquez Osuna; y a los estudiantes de la Escuela Superior de Ciencias Marinas:.

Eduardo Millán Núñez, Josué Alvarez Borrego, Esthela Elsie Millán de Alvarez y Elsa Gil de Gaxiola, su colaboración en los muestreos.

Agradecemos además la colaboración de todo el presonal del CICESE que apoyó la realización del trabajo. Nuestro especial reconocimiento al espíritu de colaboración del Sr. Alfonso Vela y del Sr. Antonio Novelo por permitirnos utilizar sus moteles en San Quintin $y$ el Estero de Punta Banda, respectivamente, a manera de laboratorio y dormitorios.

\section{BIBLIOGRAFIA.}

Acosta Ruíz, M. J. y S. Alvarez Borrego. 1974. Distribución superficial de algunos parámetros hidrológicos físicos y químicos, en el Estero de Punta Banda, B. C., en otoño e invierno. Ciencias Marinas (Mex), Vol. I (1):16-45.

Alvarez Borrego, S., J. R. Lara Lara y M. J. Acosta Ruíz. 1977. Parámetros relacionados con la productividad orgánica primarla en dos antiestuarios de Baja California. Ciencias Marinas (Mex), Vol. 4 (1): 12-22.

Chávez de Nishikawa, A. G. y S. Alvarez Borrego. 1974. Hidrología de Bahía San Quintín en invierno y primavera. Ciencias Marinas (Mex), Vol. 1 (2): 31-62.

Dawson, E. Y. 1951. A further study of upwelling and vegetation along Pacific Baja California, Mexico. Jour. Mar. Res. Vol. 10, No. 1: 39-58. 
Denman, K. L.and T. Platt. 1975. Coherences in the horizontal distributions of phytoplankton and temperature in the upper ocean. Mem. Soc. Roy. Scien. Liege, 6th series. 8:19-30.

Glooschenko, W. A., H. Curl Jr. and L. F. Small. 1972. Diel periodicity of chlorophyll a concentration in Oregon coastal waters. J. Fish. Res. Bd. Canada 29:1253-1259.

Millán Núñez, R. y S. Alvarez Borrego. 1978. Ecuaciones espectrofotométricas tricromáticas para la determinación de clorofilas a, b y c y sus feofitinas. Ciencias Marinas (Mex), Vol. 5 (I): 47-55.

Platt, T. 1972. Local phytoplankton abundance and turbulence. Deep-Sea Res. 19: 183-187.

Platt, T. 1975. Analysis of the importance of spatial and temporal heterogeneity in the estimation of annual production by phytoplankton in a small, enriched, marine basin. J. Exp. Mar. Biol. Ecol. Vol. 18:99-109.

Riley, J. P. and T. R. S. Wilson. 1967. The pigments of some marine phytoplankton species. J. Mar. Biol. Ass. U. K. 47. 351-362.

Strickland, J. D. H. 1968. A comparison of profiles of nutrient and chlorophyll concentrations taken from discrete depths and by continuous recording. Limnology and Oceanography. Vol. 13:388-391.

Shoaf, W. T. and B. W. Lium. 1976. Improved extraction of chlorophyll $a$ and $b$ from algae using dimethyl sulfoxide. Limnology and Oceanography. Vol. 21, No. 6: 926-928.

Zertuche González, J. A. y S. Alvarez Borrego. 1978. Series de tiempo de variables fisicoquimicas en las bocas de dos antiestuarios de Baja California. Ciencias Marinas (Mex), Vol. 5 (1):91-103. 